\title{
Titre and combination of ICA and autoantibodies to glutamic acid decarboxylase discriminate two clinically distinct types of latent autoimmune diabetes in adults (LADA)
}

\author{
T. Lohmann ${ }^{1}$, K. Kellner ${ }^{1}$, H.-J. Verlohren ${ }^{2}$, J. Krug ${ }^{3}$, J.Steindorf ${ }^{3}$, W.A.Scherbaum ${ }^{4}$, J.Seissler ${ }^{4}$ \\ ${ }^{1}$ Department of Internal Medicine III, University of Leipzig, Leipzig, Germany \\ ${ }^{2}$ Diabetes Speciality Practice, Leipzig, Germany \\ ${ }^{3}$ City Hospital Leipzig, Leipzig, Germany \\ ${ }^{4}$ German Diabetes Research Institute, University of Düsseldorf, Düsseldorf, Germany
}

\section{Abstract}

Aims/hypothesis. This study aimed to define the immunological parameters which could be used to identify patients with the distinct metabolic features of adult latent autoimmune diabetes.

Methods. Sera of 312 patients with short-term diabetes (duration $<5$ years) over 35 years of age at diagnosis were screened for ICA, GAD- and IA2-Ab by antibody assays validated in workshops. The antibody status was correlated with age, BMI, residual beta-cell function, measured by fasting C-peptide, onset of diabetes-related complications and markers of the metabolic syndrome (hypertension and hyperlipidaemia).

Results. A total of 51 antibody positive patients were identified. These patients had lower fasting C-peptide and less neuropathy and hypertension compared with matched antibody-negative patients. However, only patients with two or more antibodies had reduced re- sidual beta-cell function compared with antibody-negative or single antibody-positive (ICA or GAD-Ab only) patients. Patients with two or more antibodies were also leaner and had diabetes-related complications or hypertension less frequently than single antibody-positive or antibody negative-patients. IA 2 antibody status did not substantially contribute to the diagnosis or differentiation of LADA patients.

Conclusion/interpretation. We concluded that the combination of ICA and GAD antibodies and high titre of GAD antibodies are characteristic of patients with insulin deficiency with the clinical features of Type I (insulin-dependent) diabetes mellitus (LADA-type 1). Single antibody positivity and low titre antibodies are markers for LADA-type 2 associated with the clinical and metabolic phenotype of Type II (non-insulin-dependent) diabetes patients. [Diabetologia (2001) 44: 1005-1010]

Keywords GAD-Ab, ICA, diabetes mellitus, LADA.
Type I (insulin-dependent) diabetes mellitus is caused by specific autoimmune destruction of pancreatic islet cells [1]. Distinct islet cell-specific autoantibodies $(\mathrm{Ab})$, including ICA 16 as well as autoantibodies to insulin, glutamic acid decarboxylase (GAD) 65 or the tyrosine phosphatase IA2, are

Received: 15 January 2001 and in revised form: 4 May 2001

Corresponding author: T.Lohmann, Department of Internal Medicine III, University of Leipzig, Ph.-Rosenthal-Str. 27, 04103 Leipzig, Germany, E-mail: loht@medizin.uni-leipzig.de Abbreviations: Ab, autoantibodies; GAD, glutamic acid decarboxylase; ICA, islet cell antibodies; LADA, latent autoimmune diabetes in adults markers for this autoimmune process [2-5]. The GAD- and IA2-Ab are the main autoantibodies found in classic Type I diabetes with onset in childhood, adolescence and young adulthood and cover a great proportion of ICA specificities in this age group $[6,7]$. Therefore, screening for GAD- and IA2-Ab could help predict and diagnose Type I diabetes in young patients. There is also a subgroup of patients with Type I diabetes according to WHO and ADA classification [8] is who have onset in later adulthood ( $>35$ years). This is called latent autoimmune diabetes in adults (LADA) $[9,10]$ or slowly progressive Type I diabetes [11]. Patients are characterized by a similar clinical presentation to Type II diabetes at the onset of the disease and insulin requirement with- 
Table 1. Characterization of patients

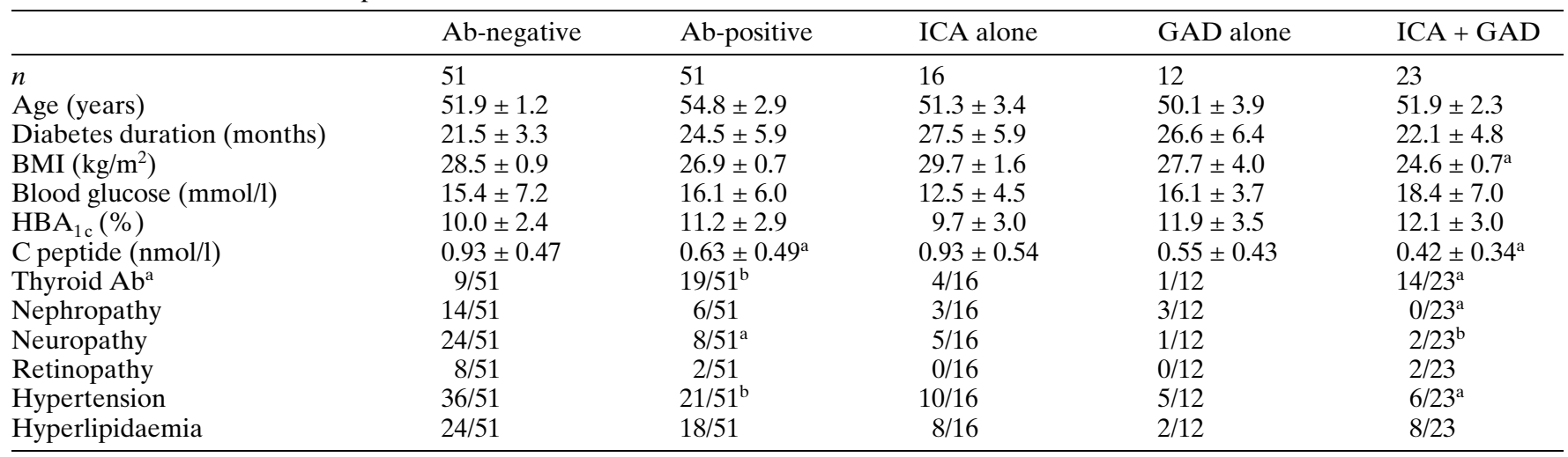

${ }^{\mathrm{a}} p<0.005$ compared with Ab-negative patients by chi square test corrected for the number of factors analysed, $n=5 ;{ }^{\mathrm{b}} p<0.05$

in a few years after diagnosis [8]. As with Type I diabetes, GAD-Ab and ICA were found in patients with LADA, while insulin- and IA2-Ab seem to have little additional diagnostic value in this age group [11-17]. Furthermore, single antibody positivity, for example for GAD-Ab and ICA, is a more common finding in LADA patients than it is in younger Type I diabetes patients $[11,15]$. In the largest study investigating islet cell-specific autoantibodies in adult patients as predictors for insulin deficiency in diabetes mellitus, the United Kingdom Prospective Diabetes Study, both GAD-Ab and ICA were predictive for the development of insulin requirement during the follow-up of 6 years [18].

In Type I diabetes of young patients, the detection of high titre or multiple islet cell-specific autoantibodies improves the diagnostic specificity [19, 20, 21]. In the older age group the importance of the titre and the number of islet cell-specific autoantibodies is less well recognized. Some studies suggest high titre of GAD-Ab $[12,17]$ or a combination of GAD-, IA2- and insulin-Ab [17] as better predictors for future insulin requirement in patients with adult Type I diabetes. Furthermore, it is still not clear whether LADA represents just a slowly progressive form of Type I diabetes or is a clinical entity of its own ("Type 1.5 diabetes") [12]. Several studies show substantial differences in the rate of progression to insulin deficiency in patients who fulfil the criteria of LADA. However, there is little information on clinical and metabolic criteria such as diabetes-related complications or associated hypertension and hyperlipidaemia as markers of the metabolic syndrome in LADA patients $[12,22]$.

This study describes a group of 51 patients of a Caucasoid German population (at low to moderate risk for Type I diabetes) classified as suffering from LADA by the presence of ICA, GAD- and IA2-Ab positivity or both. Autoimmune markers were correlated to fasting C-peptide as a marker for residual beta-cell function and clinical parameters commonly used for the classification of Type I and II diabetes in adulthood e.g. BMI, age, onset of diabetes-related complications and markers of the metabolic syndrome.

\section{Subjects and methods}

Subjects. We tested 312 consecutive sera from patients over 35 years of age at diagnosis and who developed early insulin dependency less than 5 years from diagnosis. These patients were selected by the Outpatients Department of the Department of Internal Medicine III, University of Leipzig, the City Hospital Leipzig and a practice specialising in diabetes at Leipzig. The selection of patients was based on clinical parameters. Patients with ketonuria at diagnosis were excluded. The BMI and reduced fasting C-peptide were not used as recruitment criteria. Similarly, initial insulin independency was not used as selection criterion because the decision for treatment was at the discretion of the physician in caring for the individual patient. All patients were insulin treated and metabolically fairly well controlled $\left(\mathrm{HBA}_{1 \mathrm{c}}<7.5 \%\right)$ from diagnosis to the time of investigation. From these patients, 51 sera were found positive for at least one of the following islet cell-specific autoantibodies: ICA, GAD- and IA2-Ab. A group of 51 Ab-negative patients from the initial cohort, matched for age and sex, served as the control group. These 51 Ab-negative patients were all insulin treated from diagnosis to the time of investigation and had similar amounts of blood glucose and $\mathrm{HBA}_{1 \mathrm{c}}$ at the time of diagnosis (Table 1). Therefore, both patient groups (Ab-negative and Ab-positive) were well matched for the degree of hyperglycaemia at diagnosis and during treatment up to this investigation. The patients were assessed for diabetesrelated complications such as diabetic neuropathy (vibration fork test), nephropathy (microalbuminuria in 24-h urine) and retinopathy (ophthalmologic examination). There were no differences in the frequency of complications between study centres. Furthermore, hypertension (defined as RR $>150 / 90$ or treatment with antihypertensive drugs) and hyperlipidaemia (defined as fasting cholesterol $>5.2 \mathrm{mmol} / \mathrm{l}$, fasting triglycerides $>1.7 \mathrm{mmol} / \mathrm{l}$ or treatment with antilipaemic drugs) were analysed as markers of a metabolic syndrome. The clinical data of patients are summarized in Table 1 . 
Antibody measurements. Cytoplasmic ICA's were detected by the indirect immunofluorescence test on unfixed cryostat sections of human pancreas from an organ donor with blood group 0 as described [11]. The cut-off of our assay was 5 JDFU. In the Immunology of Diabetes Workshop (IDW) ICA Proficiency Programme, our laboratory achieved values of $100 \%$ for sensitivity, specificity, validity, and consistency (Lab ID \#116). Autoantibodies to full-length GAD65 (GAD-Ab) and the intracytoplasmic domain of the tyrosine phosphatase-like protein IA-2 (IA-2-Ab) were determined by radioligand assays as described previously [11]. Amounts of antibody were expressed in arbitrary units with a cut-off of 6.5 GAD-Units and 3.4 IA-2-Units, respectively (99th centile of normal controls). ICA levels of $20 \mathrm{JDF}-\mathrm{U}$ and more and GAD-Ab levels over 20U in our assay were arbitrarily set as high titre levels. In the Combined Autoantibody Workshop, the adjusted diagnostic sensitivity for the GAD- and IA-2-Ab assays was $81 \%$ and $74 \%$ with a specificity of $99 \%$ for both assays [21]. Furthermore, we measured thyroid-specific autoantibodies (TSH-receptor- and TPO-antibodies) as a marker for polyendocrine autoimmune syndrome in all $51 \mathrm{Ab}$-positive and the 51 matched Ab-negative diabetic patients by commercially available RIA's (Brahms Diagnostica, Berlin, Germany).

Statistical analyses. In a first analysis we compared Ab-negative with Ab-positive patients and in a second analysis Ab-negative patients with groups of patients with different antibody status (ICA + GAD-Ab positive, single ICA or GAD-Ab positive) by Mann-Whitney U-test (BMI, C-peptide, age) and Fisher's Exact or chi square test with correction for the number of factors analysed as indicated. A value of $p<0.05$ was regarded as statistically significant.

\section{Results}

Characterization of autoantibody profiles in LADA patients. Of $51 \mathrm{Ab}$-positive patients, 39 (76\%) were positive for ICA, $35(68 \%)$ had GAD-Ab, and 6 (11\%) had IA-2-Ab. Altogether, 23 (45\%) of the patients had two or more antibodies, $16(31 \%)$ were only ICA positive and a further $12(23 \%)$ patients were single GAD-Ab positive. None of the sera were positive for IA2-Ab only. Of the sera with multiple antibodies, $17(33 \%)$ had GAD-Ab and ICA and 6 $(12 \%)$ had ICA, GAD- and IA2-Ab. The distribution of autoantibodies in all 51 sera is shown in Figure 1.

In the single ICA-positive group of patients, 10 had low titre $(<20$ JDF-U) and 6 had high titre (20 JDF-U and more) ICA. In the single GAD-Ab positive group, 6 had low titre $(<20 \mathrm{U})$ and 6 high titre ( $>20 \mathrm{U})$ GAD-Ab. Among patients with multiple autoantibodies, 18 (78\%) had high titre ICA and 20 $(87 \%)$ had high titre GAD-Ab. Therefore, single ICA rather than ICA in combination was more frequent for low titre ( $p<0.02$, Fisher's Exact test). Similarly, single GAD-Ab was associated with low titre more often than two or more antibody positive sera $(p<0.025$, Fisher's Exact test). Of the patients with multiple antibodies, only two $(9 \%)$ had a low titre of both ICA and GAD-Ab. Of the six patients with IA2-Ab, four had high titre IA2-Ab ( > 20U) and all

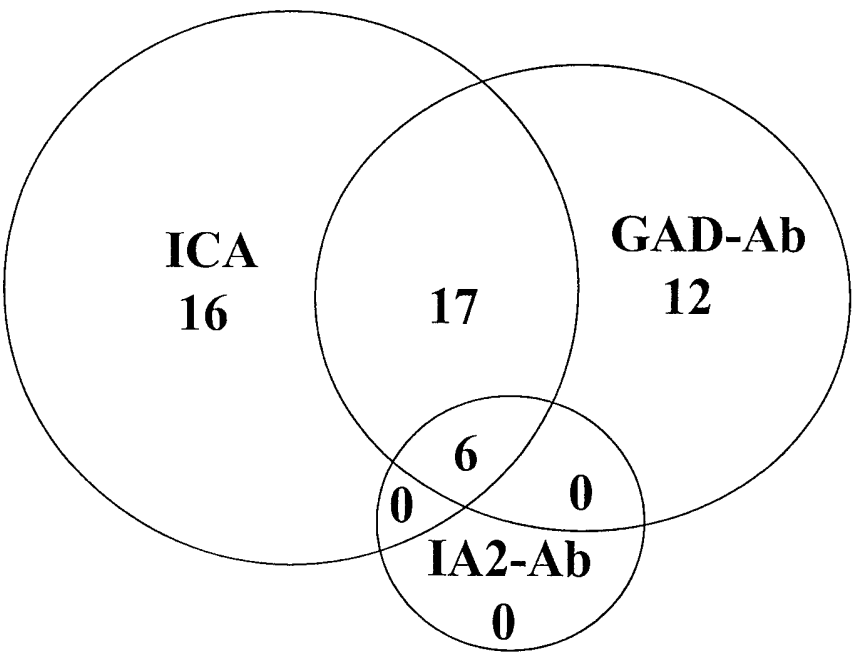

Fig. 1. Distribution of ICA, GAD- and IA2-Ab in 51 Ab-positive LADA patients

six patients with IA2-Ab had also high titre ICA and GAD-Ab or both.

Furthermore, we analysed thyroid-specific autoantibodies (TSH-receptor- and TPO-Ab) as markers for a broader polyendocrine disease in the $51 \mathrm{Ab}$-positive and the matched $51 \mathrm{Ab}$-negative patients. A total of 19 of 51 Ab-positive patients had thyroid antibodies compared to 9 of 51 Ab-negative patients $(p<0.05$, Table 1$)$. The highest frequency of thyroid antibodies was found in the group with multiple islet cell antibodies (14/23 compared to 9/51 in Ab-negative patients, $p<0.001$, Table 1$)$.

Correlation of the autoantibody status with residual beta-cell function. Overall, the Ab-positive patients had less fasting C-peptide than Ab-negative patients $(p<0.01$, Table 1, Fig. 2$)$. When the patients were subdivided according to the number of positive antibodies, multiple antibodies were associated with lower C-peptide values compared with Ab-negative patients $(p<0.002)$ and patients with single ICA $(p<0.005$, Table 1, Fig. 2$)$. In contrast, single antibody-positive patients did not differ in fasting C-peptide from antibody-negative patients. To further differentiate between patients, we investigated whether patients with high titre single antibodies (either ICA or GAD-Ab) differed from antibody-negative patients. Although the number of patients with single antibodies was limited ( $n=6$ for ICA with or above 20 JDF-U and GAD > 20 U, respectively) we observed that patients with a high level GAD-Ab had, as a trend, lower fasting $\mathrm{C}$-peptide compared with Ab-negative patients $(p<0.05$, Table 1, Fig. 2$)$ whereas high levels of single ICA-positive patients had similar fasting C-peptide values as observed in Ab-negative patients (Table 1, Fig. 2).

In summary, patients with multiple antibodies and patients with high levels of single GAD-Ab had a re- 


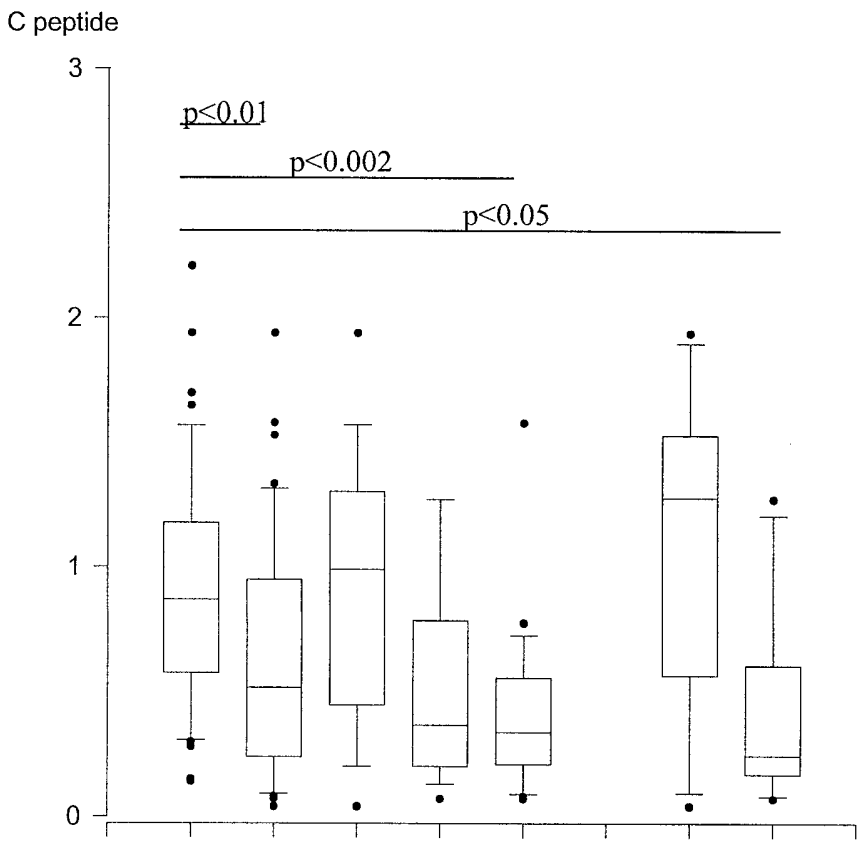

Ab-neg Ab-pos ICA GAD ICA+GAD

ICA $>20 \quad G A D>20$

Fig. 2. Fasting $\mathrm{C}$-peptide (nmol/L) values in $51 \mathrm{Ab}$-negative, $51 \mathrm{Ab}$-positive and 16 single ICA, 12 single GAD-Ab and 23 GAD- and ICA positive LADA patients. C-peptide levels of patients with single high GAD ( > 20U) and single ICA above $20 \mathrm{JDF}-\mathrm{U}$ are shown separately. Data are shown as box plots (comprising $25^{\text {th }}$ to $75^{\text {th }}$ centiles) with mean and $10^{\text {th }}$ and $90^{\text {th }}$ centiles. Points show single values outside these centiles. The $p$ values by U-test (two-tailed) are indicated.

duced residual beta-cell function compared with antibody-negative subjects, patients with single ICA and patients with low level GAD-Ab.

Correlation of autoantibodies with clinical features. Correlation of age and duration of diabetes revealed that patients with or without antibodies and with single ICA, GAD-Ab or multiple antibodies did not differ according to age or duration of diabetes (Table 1). In addition, there was no difference in BMI between the overall group of antibody-positive subjects, single GAD-Ab positive, single ICA-positive patients and the antibody-negative patients $(p>0.05$ for all groups, Table 1). However, patients with multiple antibodies had significantly lower BMI compared to Ab-negative patients $(p<0.005)$, patients with single ICA $(p<0.002)$ or single GAD-Ab $(p<0.02$, Table 1) indicating a Type I diabetes-like phenotype.

Antibody-positive patients also had fewer markers of the metabolic syndrome. Hypertension was more frequent in antibody-negative patients (36/51 compared with $21 / 51$ patients, $p<0.025$, Table 1$)$. This relation was mainly mediated by the negative association between hypertension and double antibody positivity. Only 6 of 23 of the patients with both ICA and GAD-Ab positive had hypertension compared with
36 of 51 Ab-negative patients $(p<0.005)$ and 10 of 16 patients with single ICA or 5 of 12 patients with single GAD-Ab ( $p>0.05$, respectively). We found no significant differences in the frequency of hyperlipidaemia between the patient groups (Table 1).

Correlation of different LADA groups with diabetesrelated complications. There were fewer antibodypositive patients suffering from neuropathy compared with Ab-negative patients (8/51 compared to $24 / 51, p<0.001$, Table 1). This difference could be confirmed in patients who were positive for both ICA and GAD-Ab compared with Ab-negative patients $(2 / 23$ compared to $8 / 51, p<0.005)$. Single ICA (5/17 patients) or single GAD-Ab positive patients (1/12 patients) did not differ in the frequency of neuropathy from antibody-negative patients. We found nephropathy less frequently in antibody-positive patients compared with antibody-negative patients but this difference failed to reach statistical significance (6/51 compared to $14 / 51$ patients, $p>0.05)$. However, patients in whom both ICA and GAD-Ab were positive had less nephropathy compared with antibodynegative patients $(0 / 23$ compared with $14 / 51$ patients, $p<0.025$, Table 1$)$.

\section{Discussion}

Type I diabetes is a disease with onset usually in childhood and adolescence but up to a third of patients will be diagnosed in adulthood over 35 years of age [23]. For these patients, the term "latent autoimmune diabetes in adults" (LADA) has been coined $[9,10]$. LADA patients have a few immunological and genetic characteristics which are different from patients with classic Type I diabetes, such as lower frequency of HLA risk alleles (DR3/ $\mathrm{DQ}^{*} 0201, \mathrm{DR} 4 / \mathrm{DQ} * 0302$ ) or lower frequency of IA2- and insulin-Ab [15, 17, 24]. We found a subgroup of six patients with high ICA but no GADand IA2-Ab (and also no insulin-, IA2-beta- or SOX13-antibodies) in this study as described in our previous reports $[11,15]$. Therefore, an additional still not detected autoantigen could be present in some LADA patients. The immunological markers for LADA, especially GAD-Ab and ICA, point to the contribution of autoimmune phenomena in the disease. This is supported by detection of a lymphocytic infiltrate in the beta cells of a 65-year-old woman with GAD-Ab and residual beta-cell function typical of LADA [25]. However, the evaluation of clinical data and follow-up studies suggests that LADA comprises a heterogeneous population of diabetic patients throwing into doubt the hypothesis that these patients represent one clinical entity. We aimed, therefore, to define immunological criteria to identify discrimination of patients with a more aggressive 
form of beta-cell autoimmunity and separate them from those patients with a benign autoimmune form associated with Type II diabetes-like metabolic phenotype.

Indeed, we found that both multiple islet cell-specific autoantibodies and high titre GAD-Ab $(>20 \mathrm{U}$ in our assay) are associated with low fasting C-peptide values as a marker of reduced beta-cell function, decreased BMI, a low frequency of hypertension and a lower prevalence of diabetes-associated complications (LADA-Type 1). In contrast, patients with single antibodies of low titre had the same clinical and metabolic markers as antibody-negative Type II diabetes patients (LADA-Type 2). These findings are clinically important because patients with ongoing autoimmune response could stabilize residual betacell function if given early insulin therapy [27]. In our series, the clinical parameters of LADA formed the basis for selecting patients, especially in cases of early insulin dependency. However, patients with ketoacidosis at diagnosis were excluded, being classified as having classic Type I diabetes. We did not use initial insulin independency to define LADA patients because this criterion depends on the subjective decision of the physician caring for the individual patient.

The diagnosis of autoimmune diabetes in adults is mainly based on detection of islet cell-specific autoantibodies. In classic Type I diabetes, prediction of diabetes is improved by high titre antibodies or the detection of multiple antibodies suggesting that these markers indicate a rapid destruction of beta cells [19, 20]. We show here that this could also be true for patients with LADA. Furthermore, patients with multiple islet cell autoantibodies could have a broader form of polyendocrine autoimmune disease because they also more often have thyroid autoantibodies compared with islet-cell antibody-negative or single autoantibody-positive patients. In agreement with a former study using ICA [27], we found that multiple or high titre antibodies also appear in some obese patients so that being overweight does not as a rule exclude LADA. Of note, patients with low titre antibodies have a clinical picture similar to that found in Type II diabetes. Prospective studies are necessary to clarify whether these antibodies indicate beta-cell destruction or can also be induced in patients with cell damage due to beta-cell stress in Type II diabetes [28].

Besides the association with a low BMI and C-peptide values, patients with LADA-Type 1 had less diabetic neuropathy and nephropathy compared with antibody-negative patients. These patients therefore also resemble Type I diabetes patients who develop diabetes-related complications such as these not in the first 5 years after diagnosis but rather late. The patients with LADA-Type 2 had diabetes-related complications as frequently as antibody-negative patients. Our Ab-negative and LADA-Type 2 patients were well matched to LADA-Type 1 patients for age, sex, duration of diabetes and insulin therapy and also for metabolic control at diagnosis and thereafter (Table 1) but they could have had a longer preclinical period of silent diabetes and, therefore, a longer time of exposure to hyperglycaemia, contributing to an earlier manifestation of diabetes-related complications.

Furthermore, we analysed hypertension and hyperlipidaemia as markers of a metabolic syndrome in these patient groups. Patients with LADA-Type 1 had significantly less hypertension than antibodynegative patients and, again, patients with LADAType 2 did not differ with respect to hypertension and hyperlipidaemia from antibody-negative patients. These data confirm previous studies on long term ( $>5$ years) LADA patients showing less hypertension in antibody-positive patients compared with antibody-negative subjects $[12,21]$. However, the latter studies did not find differences in diabetes-related complications between the patient groups probably because of the longer duration of disease than in patients in our study.

In conclusion, patients with single diabetes-related antibodies have the same clinical and metabolic characteristics as Type II diabetes patients but patients with multiple antibodies have the same features as patients with Type I diabetes. We recommend the screening of ICA and GAD-Ab and the assessment of antibody levels for discrimination of patients with LADA-Type 1, who are at risk for insulin requirement, from patients with LADA-Type 2, who have characteristics more closely associated with a non-insulin-dependent form of diabetes. Measuring of IA2$\mathrm{Ab}$ does not appear to be necessary for this purpose because we found these $A b$ to be rare and never to appear alone in this patient group. Our data could contribute to a more accurate classification of LADA diabetes and could help clinicians choose an appropriate form of diabetes treatment. Our data could also be used to design intervention trials in these patients.

Acknowledgements. T.Lohmann and J.Seissler were supported by grants from the German Diabetes Association. T.Lohmann was supported by the Interdisciplinary Centre for Clinical Research at the University of Leipzig. The technical help of A. Kluge and S. Laue is gratefully acknowledged.

\section{References}

1. Atkinson MA, Maclaren NK (1994) The pathogenesis of insulin-dependent diabetes mellitus. N Engl J Med 331: 1428-1436

2. Bottazzo GF, Florin-Christensen A, Doniach D (1974) Islet cell antibodies in diabetes mellitus with autoimmune polyendocrine deficiencies. Lancet ii : 1279-1283

3. Palmer JP, Asplin CM, Clemons P et al. (1983) Insulin antibodies in insulin-dependent diabetes before insulin treatment. Science 222: 1337-1339 
4. Baekkeskov S, Aanstoot HJ, Christgau S et al. (1990) Identification of the $64 \mathrm{~K}$ autoantigen in insulin-dependent diabetes as the GABA-synthesizing enzyme glutamic acid decarboxylase. Nature 347: 151-156

5. Lan MS, Lu J, Goto Y, Notkins AL (1994) Molecular cloning and identification of a receptor-type protein tyrosine phosphatase, IA-2, from human insulinoma. DNA Cell Biol 13: 505-514

6. Seissler J, Morgenthaler NG, Achenbach P et al. (1996) Combined screening for autoantibodies to IA-2 and antibodies to glutamic acid decarboxylase in first degree relatives of patients with IDDM. Diabetologia 39: 1351-1356

7. Verge CF, Gianini R, Kawasaki E et al. (1996) Prediction of Type I diabetes in First-degree relatives using a combination of insulin, GAD, and ICA512bdc/IA-2 autoantibodies. Diabetes 45: 926-933

8. WHO/ADA (1997) Report of the Expert Commitee on the diagnosis and classification of diabetes mellitus. Diabetes Care 20: 1183-1197

9. Tuomi T, Groop LC, Zimmet PZ, Rowley MJ, Knowles W, Mackay IR (1993) Antibodies to glutamic acid decarboxylase reveal latent autoimmune diabetes mellitus in adults with a non-insulin-dependent onset of disease. Diabetes 42: 359-362

10. Zimmet PZ, Tuomi T, Mackay IR et al. (1994) Latent autoimmune diabetes mellitus in adults (LADA): The role of antibodies to glutamic acid decarboxylase in diagnosis and prediction of insulin dependency. Diabetes Med 11: 299-303

11. Seissler J, De Sonnaville JJJ, Morgenthaler NG et al. (1998) Immunological heterogeneity in type I diabetes: presence of distinct autoantibody patterns in patients with acute onset and slowly progressive disease. Diabetologia 41: 891-897

12. Tuomi T, Carlsson A, Li H et al. (1999) Clinical and genetic characteristics of Type II diabetes with and without GAD antibodies. Diabetes 48: 150-157

13. Niskanen LK, Tuomi T, Karjalainen J, Groop LC, Uusitupa MIJ (1995) GAD antibodies in NIDDM: Ten-year followup from the diagnosis. Diabetes Care 18: 1557-1565

14. Groop LC, Bottazzo GF, Doniach D (1986) Islet cell antibodies identify latent Type I diabetes in patients aged 35-75 years at diagnosis. Diabetes 35: 237-241

15. Lohmann T, Seissler J, Verlohren HJ et al. (1997) Distinct genetic and immunological features in patients with insulin-dependent diabetes below and above age 40 at onset. Diabetes Care 20: 524-529

16. Littorin B, Sundkvist G, Hagopian W et al. (1999) Islet cell and glutamic acid decarboxylase antibodies present at di- agnosis of diabetes predict the need for insulin treatment. Diabetes Care 22: 409-412

17. Kasuga A, Maruyama T, Nakamato S, Ozawa Y, Suzuki Y, Saruta T (1999) High-titer autoantibodies against glutamic acid decarboxylase plus autoantibodies against insulin and IA-2 predicts insulin requirement in adult diabetic patients. J Autoimmun 12: 131-135

18. Turner R, Stratton I, Horton V et al. (1997) UKPDS 25: autoantibodies to islet-cell cytoplasm and glutamic acid decarboxylase for prediction of insulin requirement in Type II diabetes. Lancet 350: 1288-1293

19. Bonifacio E, Bingley PJ, Shattock M et al. (1990) Quantification of islet-cell antibodies and prediction of insulin-dependent diabetes. Lancet 335: 147-149

20. Bingley PJ, Christie MR, Bonifacio E et al. (1994) Combined analysis of autoantibodies improves prediction of IDDM in islet-cell antibody-positive relatives. Diabetes 43: $1304-1310$

21. Verge CF, Stenger D, Bonifacio E et al. (1998) Combined use of autoantibodies (IA-2 autoantibody, GAD autoantibody, insulin autoantibody, cytoplasmic islet cell antibodies) in Type I diabetes. Diabetes 47: 1857-1866

22. Isomaa B, Almgren P, Henricsson M, Tuomi T, Groop L, Sarelin L (1999) Chronic complications in patients with slowly progressing autoimmune Type I diabetes (LADA). Diabetes Care 22: 1347-1353

23. Karjalainen J, Salmela P, Ilonen J, Surgel H-M, Knip M (1989) A comparison of childhood and adult Type 1 diabetes mellitus. N Engl J Med 320: 881-886

24. Horton V, Stratton I, Bottazzo GF et al. (1999) Genetic heterogeneity of autoimmune diabetes: age of presentation in adults is influenced by HLA DRB1 and DQB1 genotypes (UKPDS 43). Diabetologia 42: 608-612

25. Shimada A, Imazu Y, Morinaga S et al. (1999) T-cell insulitis found in anti-GAD65 ${ }^{+}$diabetes with residual $\beta$-cell function. Diabetes Care 22: 615-617

26. Kobayashi T, Nakanishi K, Murase T, Kosaka K (1996) Small doses of insulin as a strategy for preventing slowly progressive $\beta$-cell failure in islet cell antibody-positive patients with clinical feature of NIDDM. Diabetes 45: 622-626

27. Gottsäter A, Landin-Olsson M, Lernmark A, Fernlund P, Sundkvist G (1994) Islet cell antibodies are associated with $\beta$-cell failure also in obese adult onset diabetic patients. Acta Diabetol 31: 226-231

28. Rolandsson O, Hägg E, Hampe et al (1999) Glutamate decarboxylase (GAD65) and tyrosine phosphatase-like protein (IA-2) autoantibodies index in a regional population is related to glucose intolerance and body mass index. Diabetologia 42: 555-559 